LAWRENCE LIVERMORE NAT IO N A L LABORATORY
Implementation of a Near Back-Scattering Imaging System on The National Ignition Facility

A.J. Mackinnon, T. McCarville, C. Niemann, K. Piston, G. Jones, I. Reinbachs, R. Costa, J. Celeste, R. Griffith, R. Kirkwood, B. MacGowan, S. Glenzer

April 16, 2004

15th Topical Conference on High Temperature Plasma Diagnostics San Diego, CA, United States April 19, 2004 through April 22, 2004 
This document was prepared as an account of work sponsored by an agency of the United States Government. Neither the United States Government nor the University of California nor any of their employees, makes any warranty, express or implied, or assumes any legal liability or responsibility for the accuracy, completeness, or usefulness of any information, apparatus, product, or process disclosed, or represents that its use would not infringe privately owned rights. Reference herein to any specific commercial product, process, or service by trade name, trademark, manufacturer, or otherwise, does not necessarily constitute or imply its endorsement, recommendation, or favoring by the United States Government or the University of California. The views and opinions of authors expressed herein do not necessarily state or reflect those of the United States Government or the University of California, and shall not be used for advertising or product endorsement purposes. 


\title{
Implementation of a near back-scattering imaging system on the National Ignition Facility.
}

\author{
A.J.Mackinnon, T.McCarville, C. Niemann, K. Piston, G. Jones, I. Reinbachs, R. Costa, \\ J. Celeste, R. Griffith, R.Kirkwood, B.MacGowan, S. Glenzer
}

Lawrence Livermore National Laboratory, Livermore, Ca 94551

\begin{abstract}
:
A near back-scattering imaging diagnostic system is being implemented on the first quad of beams on the National Ignition Facility. This diagnostic images diffusing scatter plates, placed around the final focus lenses on the NIF target chamber, to quantitatively measure the fraction of light back-scattered outside of the incident cone of the focusing optics. The imaging system consists of a wide-angle lens coupled to a gated CCD camera, providing $3 \mathrm{~mm}$ resolution over a $2 \mathrm{~m}$ field of view. To account for changes of the system throughput due to exposure to target debris the system will be routinely calibrated in situ at $532 \mathrm{~nm}$ and $355 \mathrm{~nm}$ using a dedicated pulsed laser source. The diagnostic will be described and recent results will be presented. Work performed under the auspices of the U.S. Department of Energy by UC/Lawrence Livermore National Laboratory under Contract No. W-7405-ENG-48.
\end{abstract}




\section{Introduction}

The National Ignition Facility uses two diagnostics to study the laser coupling interaction in inertial fusion targets: the Full Aperture Back Scatter diagnostic (FABS), and the Near Backscatter Imaging diagnostic (NBI). Both diagnostics measure the laser energy backscattered from targets. An NBI system was used to measure light backscattered outside the cone angle of the focusing optics [1]. On the Nova laser at LLNL, this diagnostic showed that under certain circumstances it is possible for an appreciable fraction of the incident laser light to be back-scattered outside the focusing lens. Calculations with the laser-plasma simulation code F3D have also predicted that substantial light can be scattered outside the final NIF lens for long plasma scalelengths that will be required for ignition scale hohlraums. Presently this fraction of light is not measured by the FABS system and so must be measured by an NBI system to test the code predictions and to fully characterize the laser plasma interaction.

The entrance aperture for four laser beams is shown in Figure 1(a) as viewed from chamber center. As an illustration of light distribution an F3D calculation of the laser light scattered back from a gas filled target toward this aperture is also shown in figure 1(b) (it should be noted that the contrast for this figure has been enhanced to make the light outside the lens more visible). For this particular calculation, which has no smoothing, the backscatter fraction was in the region of $20 \%$, with half of this energy outside the final focus lens. It is clear that in these circumstances the FABS diagnostic, described in a companion paper, will only be able measures some of the energy scattered directly back into the aperture of incoming beams. Only the FABS and NBI diagnostics together provide the tool required to verify laser target interaction models.

The NBI diagnostic consists of two subassemblies shown in Figure 2. Laser light backscattered from the target is incident on a $2 \mathrm{~m} \times 2 \mathrm{~m}$ scatter plate located around the chamber wall laser entrance aperture. The scatter plate has four cut-outs to allow passage of incoming beams. Laser light scattered by the target strikes the scatter plate, which scatters light within the view of imaging cameras outside the chamber. The images of backscattered laser light are compared to calibration images taken when the scatter plate is illuminated with a known energy source. The backscattered energy is measured by comparing target shot images to calibration images. 
Up to $20-30 \%$ of the incident energy may be back scattered from some targets. The NBI diagnostic uses two separate cameras to measure the energy from Raman and Brillouin scattering processes separately. Brillouin scattered light has a bandwidth around a few nanometers of the incident $351.5 \mathrm{~nm}$ laser beams, and the camera response is essentially constant over this wavelength range. Raman scattered light may fall anywhere between $400-800 \mathrm{~nm}$, depending on the target, and camera response varies significantly over this bandwidth. Hence, a relative spectral measurement of the Raman scattered light is also required to determine Raman backscatter energy.

\section{The Imaging Subassembly}

The NBI diagnostic uses intensified cameras to image the scatter plate. The intensifier captures an image of light scattered off the scatter plate while blocking both direct illumination from the target and multiple reflections inside the chamber. An intensifier gate width of $10 \mathrm{~ns}$ is short enough to block direct target illumination and multiple scattering from the chamber, and long enough to capture the 1-3 ns pulse from the scatter plate. A variety of commercial intensified cameras were tested for this application. The response curves for two units tested are shown in Figure 3 for a $10 \mathrm{~ns}$ gate and $351 \mathrm{~nm}$ light. A relatively flat response function over the gate is desired, and this criterion was used to select the NBI cameras. The dynamic range and useful range of intensifier gain were also measured for various intensified cameras. A dynamic range of 200 was typical, and the responses were linear for gains up to 200. While high dynamic range is advantageous in our application, gain linearity is not as critical because there are many photons available, and the gain will be fixed to a modest value.

The two intensified cameras are packaged in a light tight enclosure located outside the target chamber. A design model of the enclosure assembly, with covers removed, is shown in Figure 4. Filters located at the enclosure entrance select a wavelength range for each camera and attenuate the light intensity. The remaining light reflects off a turning mirror and passes through a focusing lens on its way to the camera

intensifier cathode. For high target backscatter shots, attenuation up to $10^{8}$ is required to avoid camera saturation. For calibration, some of the ND filters are removed to maximize the camera signal. 
Target back-scatter is captured by turning the camera intensifiers on at a prescribed time relative to a fiducial signal generated by the main laser. The fiducial signal is referenced to the time laser light arrives at chamber center. The fiducial signal and intensifier gate monitor pulses are both recorded by an oscilloscope, and relative timing is adjusted to achieve the required temporal separation. Using the fiducial, proper timing can be achieved without firing a high power laser pulse. To verify timing has been achieved during a laser pulse, an ET-2000 diode collects light from the scatter plate, and the diode trace is recorded along with the fiducial and monitor pulses.

\section{The Scatter Plate Subassembly}

The challenge of the scatter plate design is to select materials compatible with the environment. The scattering material must be wavelength independent, suitable for vacuum, and have high scattering efficiency. A number of materials meet these requirements. The main challenge is that the scatter plate also must not damage when subjected to up to $1 \mathrm{~J} / \mathrm{cm}^{2}$ backscatter of $351 \mathrm{~nm}$ light. The scatter plate and window must last for hundreds of shots before accumulating noticeable damage. Damage tests with opal and etched glass show they damage at $0.5 \mathrm{~J} / \mathrm{cm}^{2}$ of $351 \mathrm{~nm}$ light [4], ruling out glass scattering materials.

Spectralon, a product made by Labsphere, is a commonly used scatterer for optics applications. Its scattering properties are well characterized and available on the Labsphere web site. Spectralon was tested at LLNL for laser damage with $351 \mathrm{~nm}$ light, and found to be acceptable for use at $1 \mathrm{~J} / \mathrm{cm}^{2}$. The results are shown in Table 1 . Spectralon is a low density form of PTFE Teflon. Commercial grade PTFE was damage tested for comparison, and significant damage was visible at $1 \mathrm{~J} / \mathrm{cm}^{2}$.

The linearity of Spectralon vs. incident energy density has also been independently tested using a 200ps doubled YLF laser at LLNL. and compared with both Aluminum and Teflon. The results, shown in figure 5, show that Spectralon provides a linear reflectance for energy density up to $3 \mathrm{Jcm}-2$ at $527 \mathrm{~nm}$. Further tests are underway to measure the $355 \mathrm{~nm}$ response. Based on these results, Spectralon was chosen for the scatter plate. Target debris and shrapnel pose another challenge for the scatter plate. Debris accumulates on the scatter plate in the form of powdered oxides and metal 
droplets. Estimations of the accumulation rate from no-yield targets during initial NIF operation at about 20 nanograms $/ \mathrm{cm}^{2}$ per shot, or about 20 angstroms thickness per shot if deposited uniformly [3]. Over time the debris builds up and absorbs light, reducing scattering efficiency and changing the scatter plate calibration.

To estimate the reduction in scattering efficiency from debris, stainless steel was laser ablated onto Spectralon at various deposition thicknesses and the scattering efficiency was measured. The results indicate the scatter plate should be re-calibrated about once per month. This duration is acceptable because calibration can be done quickly while the chamber remains under vacuum. After a few months it will be necessary to clean debris off the scatter plate to recover high efficiency over the whole wavelength range. Debris is easily removed if a smooth transparent window is placed on top of the Spectralon. If the debris is loose, it can simply be wiped away. If it is adherent, it can be removed by replacing window panels. Fused silica is an obvious choice for the window because of its high damage threshold. But fused silica is somewhat expensive, and the low fracture toughness of glass could produce spallation fragments from high velocity target shrapnel. Spallation fragments would be a threat to the final focusing optics directly underneath the scatter plate.

A plastic would reduce the tendency to produce spallation fragments, but most are not transmissive to $351 \mathrm{~nm}$ light. However, a few acrylics have high transmission at 351 $\mathrm{nm}$ and through the visible wavelength range. OP-4, and acrylic produced by Cryo, was selected for damage testing with $351 \mathrm{~nm}$ laser pulses. It showed little visible damage at 1 $\mathrm{J} / \mathrm{cm}^{2}$. Based on the damage test, OP-4 has been selected for the scatter plate window.

\section{Energy Calibration}

The camera and scatter plate are calibrated together in order to determine the net response to target backscatter. Laser pulses of known energy are directed onto the scatter plate, and images of the scattered light are captured from a Minilite II YAG laser manufactured by Continuum. The pulses come from a laser inside the NBI camera enclosure. A calibration function is generated from the images, and when applied to target shots this function determines the target energy back scattered. Frequency conversion crystals provide output wavelengths at 532 or $355 \mathrm{~nm}$, in $3 \mathrm{~ns}$ pulses. The 532 
$\mathrm{nm}$ wavelength is used to calibrate one camera for Raman backscatter, and $355 \mathrm{~nm}$ is used to calibrate the other for Brillouin backscatter. Each pulse contains a few $\mathrm{mJ}$ of energy spread over about a $10 \mathrm{~mm}$ spot. This provides an observable signal when all ND filters are removed from the camera view. The pulse energy is first measured with a calorimeter. Mirrors are then installed to direct calibration laser light through one of the camera viewing ports and onto the scatter plate. Only one camera is calibrated at a time because the 2 " turning mirror blocks the other cameras view. The calibration laser timing is synchronized with the camera's intensifier gate to capture the scattered light.

The last turning mirror is motorized in order to scan the scatter plate point by point with calibration pulses. Cosine corrections are made at each point to account for the small off normal angle of the calibration laser and target light on the scatter plate. A continuous calibration function is generated from interpolation of the point map. The continuous function is applied to the intensity profile captured from a target shot to calculate backscattered energy. Pulses at $355 \mathrm{~nm}$ are adequate to calibrate the few narrow bandwidth of Brillouin backscatter. For the camera observing Raman backscatter, the calibration pulse response observed at $532 \mathrm{~nm}$ pulses must be extrapolated over the Raman bandwidth. This can be done using the wavelength response curve provided by the camera manufacturer, and a relative measurement of the target shot's Raman spectra. The FABS diagnostic measures the relative spectral intensity of Raman scattered light, and this distribution is applied to the NBI image of Raman back scatter.

\section{Summary}

The NBI diagnostic is now completing its installation and test phase in NIF. The cameras are installed and fully operational, and proper timing to NIF laser pulses has been demonstrated. The scatter plate and calibration laser are ready for installation, and will be commissioned with target shots in the next few months. The FABS diagnostic is already operational and measuring the Raman and Brillouin energy backscattered down the incident laser beam path. FABS measurements have shown that some of the backscattered light falls outside the incident beam path, and should be observable by NBI. Once NBI is operational, its energy measurements will complement those in FABS 
to measure the total backscattered energy. The current NIF schedule calls for measuring total backscattered energy in the summer of 2004.

The authors gratefully acknowledge the Bechtel operations team for their valuable contribution to this project. This work was performed under the auspices of the U.S. Department of Energy by the University of California, Lawrence Livermore National Laboratory under contract No. W-7405-Eng-48.

\section{References:}

1. R. Kirkwood Rev. Sci. Instruments 1999

2. Backscatter calculations by F3D by E.Williams et al

3. Eder. D, Private commun

4. Internal memos from M. Runkel, J. McElroy, and R. Luthi, LLNL

\section{Figure Captions}

1. (a) A view of the laser beam entrance at the target chamber wall, (b) and a calculation of backscattered light intensity around the port (calculation parameters?)

2. The scatter plate is located around the entrance aperture of a beam quad, and viewed by the imaging cameras outside the chamber.

3. Sensitivity of two intensified CCD cameras for a $10 \mathrm{~ns}$ gate width and $355 \mathrm{~nm}$ light

4. Camera enclosure shown mounted to the chamber, and a cut away view illustrating the optical path.

5. The response of spectralon was determined to be linear up to a flux of $3 \mathrm{~J} / \mathrm{cm}-2$

\section{Tables}

1. Damage test results for Optical grade spectralon 
(b)
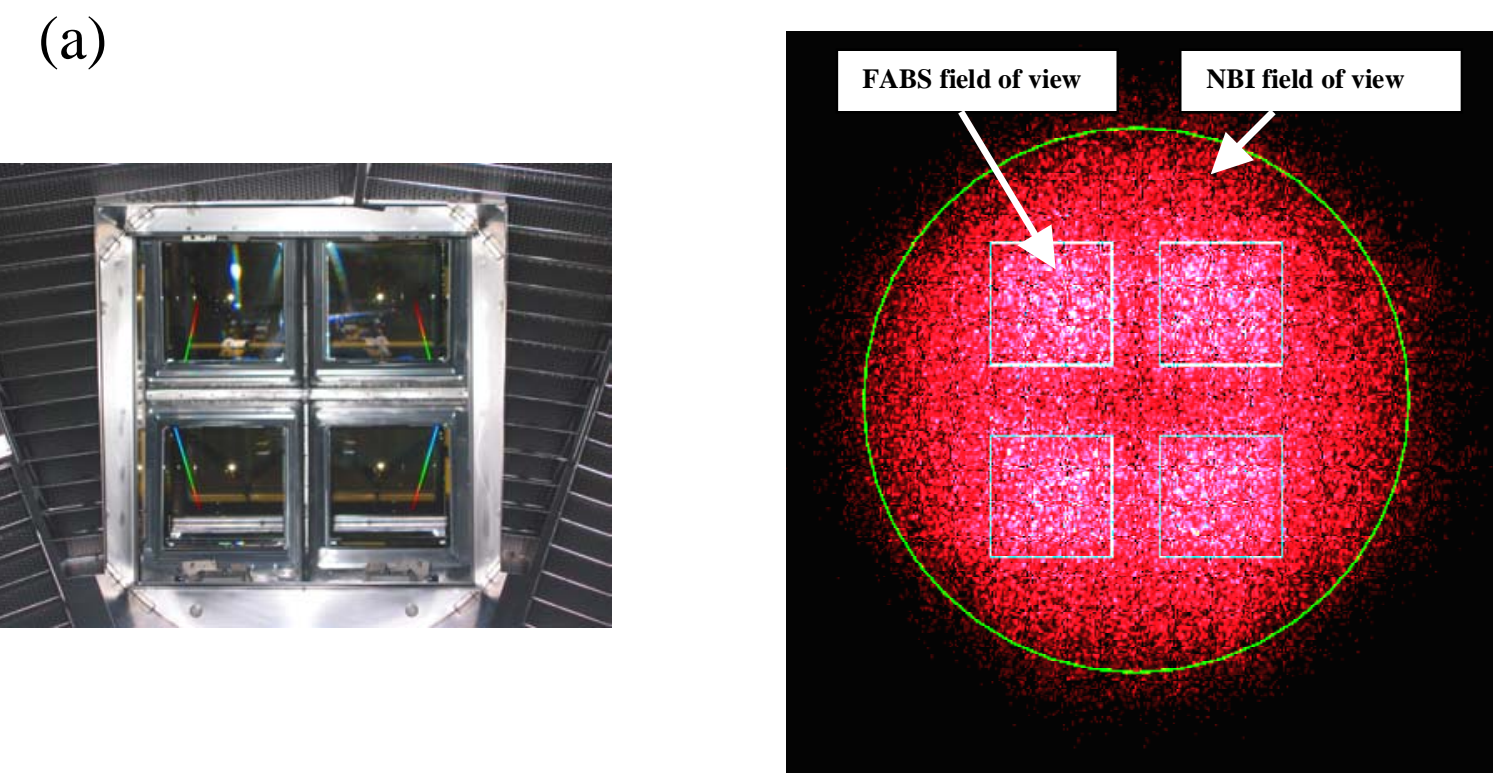

Figure 1. A view of the laser beam entrance at the target chamber wall, and a calculation of backscattered light intensity around the port (calculation parameters?) 


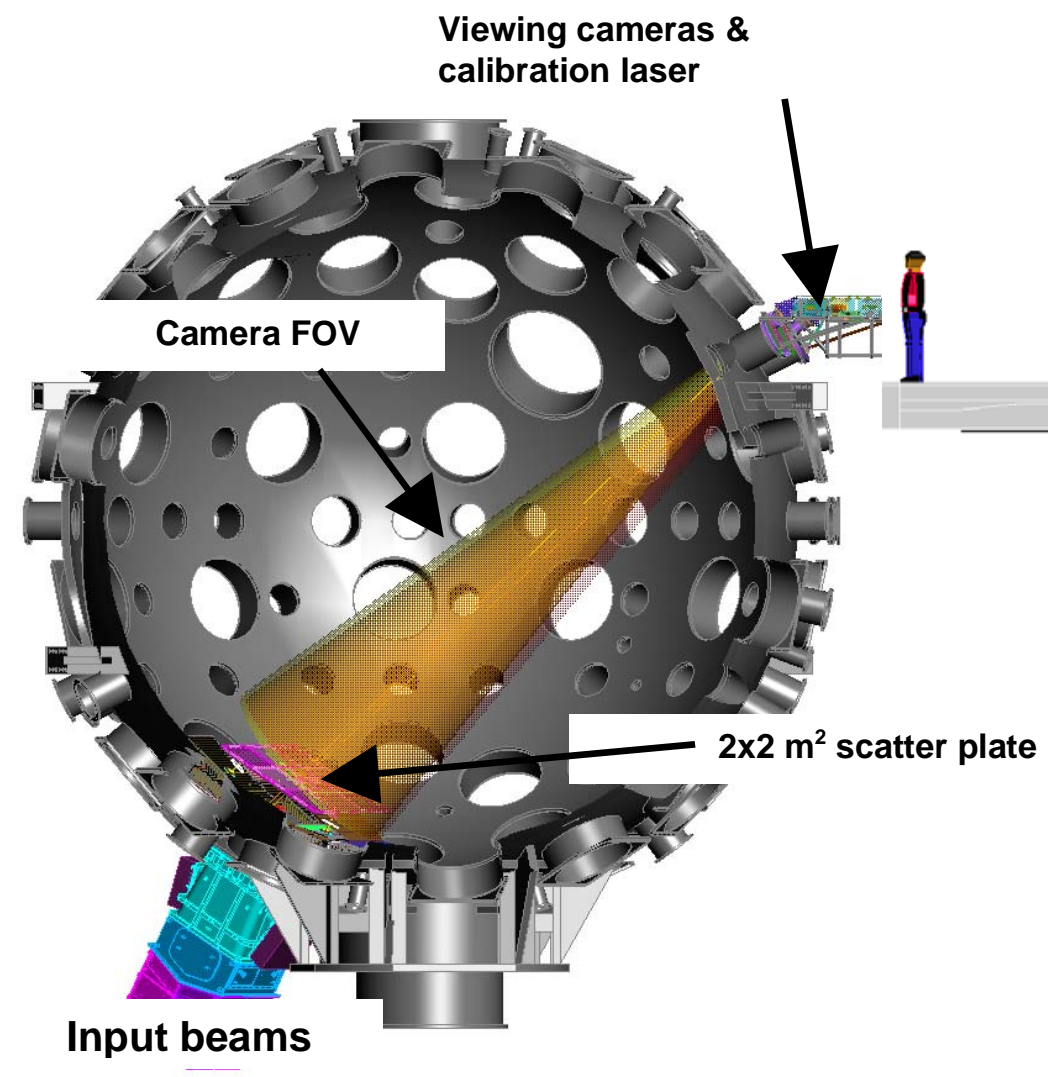

Figure 2. The scatter plate is located around the entrance aperture of a beam quad, and viewed by the imaging cameras outside the chamber. 


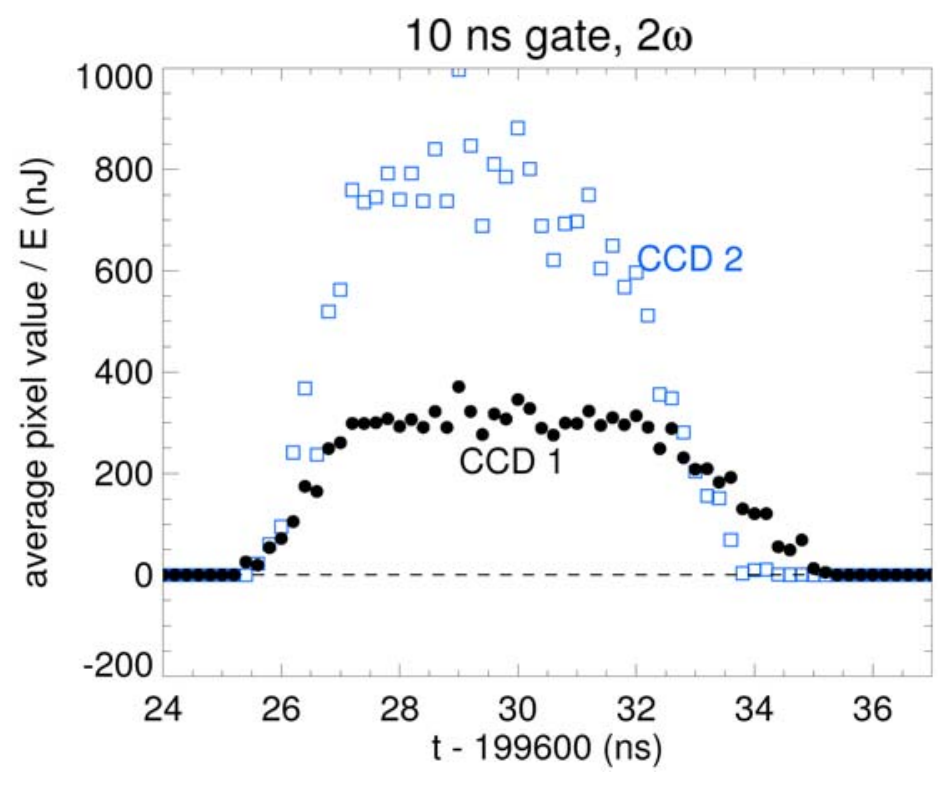

Figure 3. Sensitivity of two intensified cameras for a $10 \mathrm{~ns}$ gate width and $351 \mathrm{~nm}$ light. 


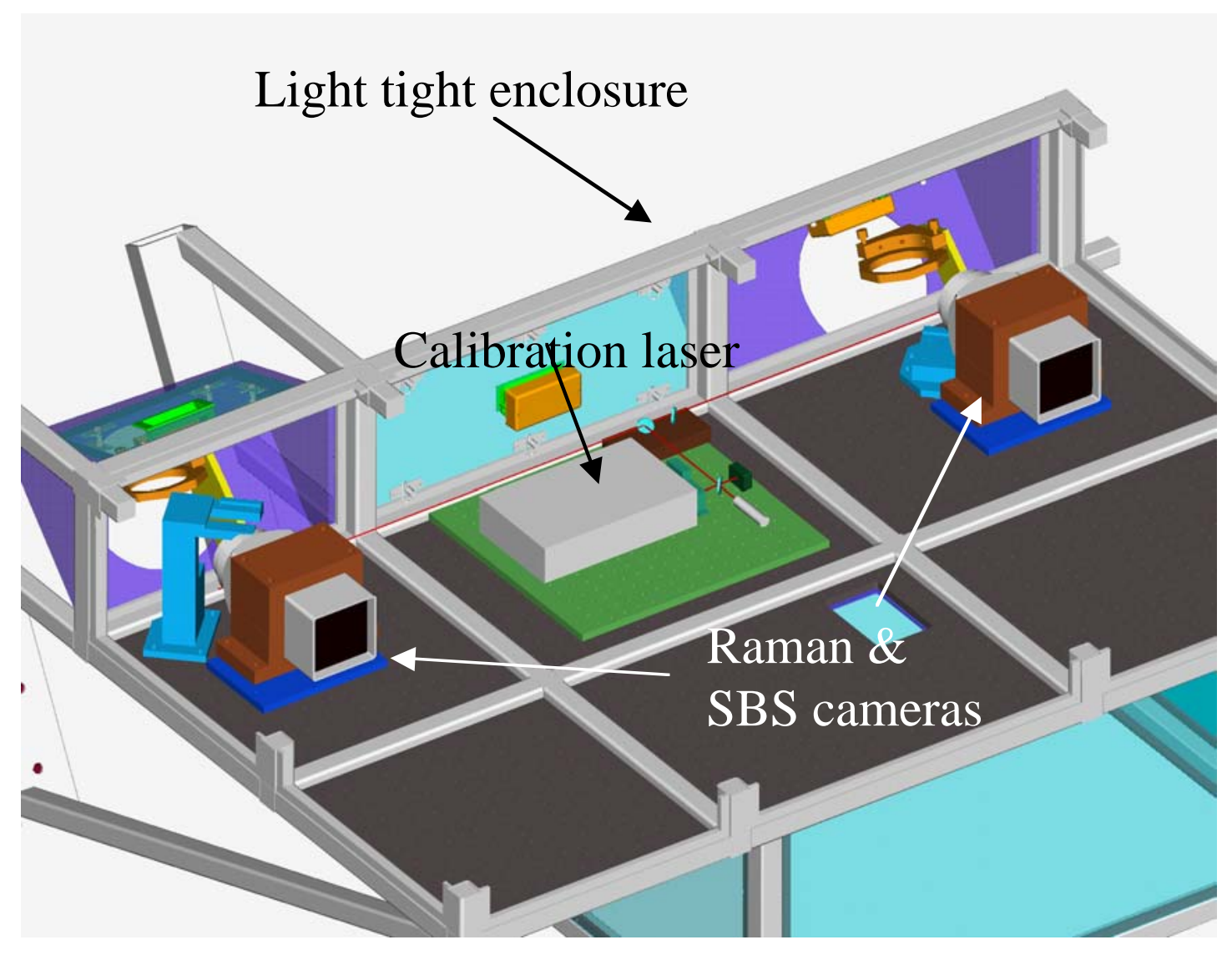

Figure 4. The gated imaging cameras and calibration laser view the scatter plate from inside a light tight enclosure 


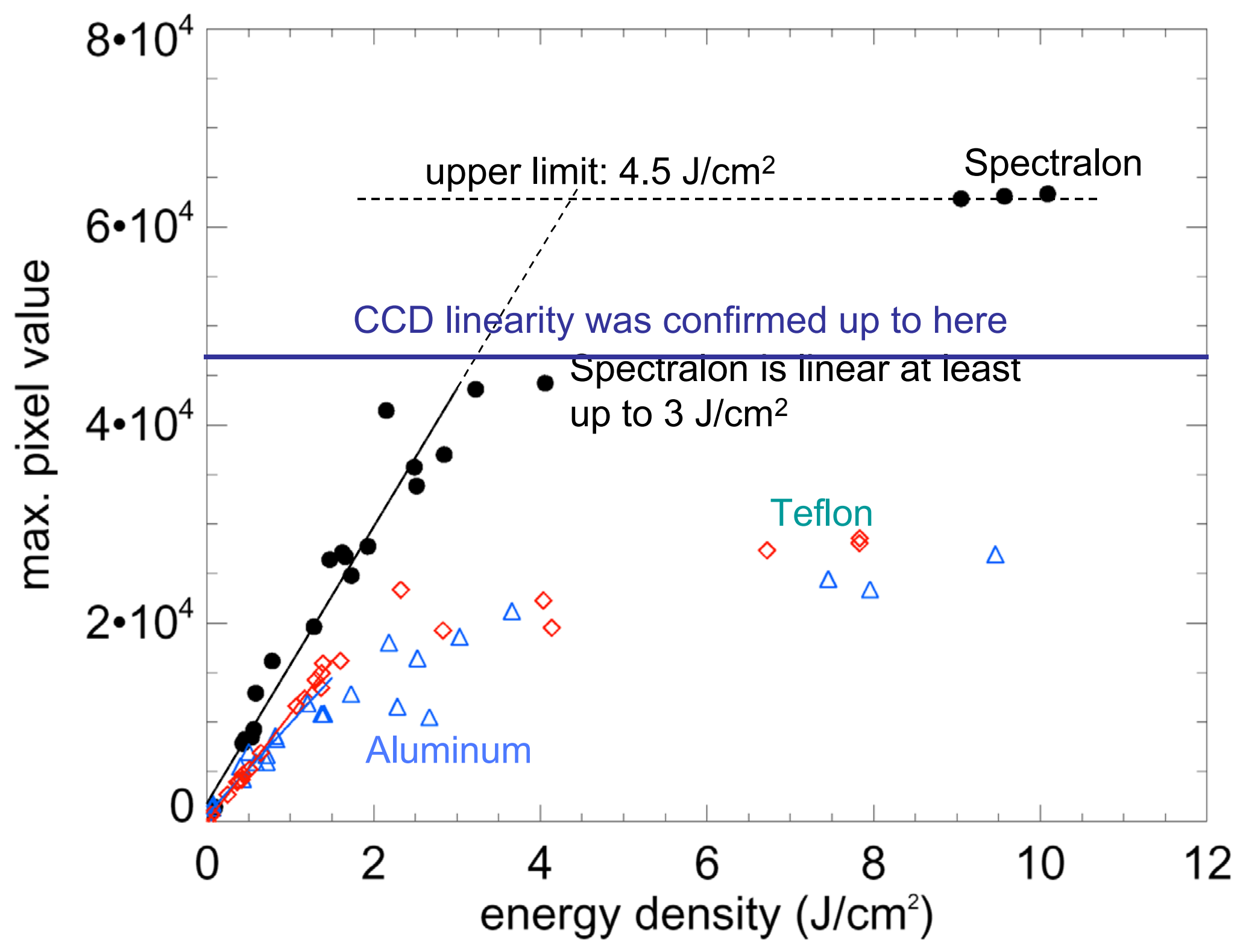

Figure 5 All three diffusers saturate at a few $\mathrm{J} / \mathrm{cm}^{2}$, spectralon has highest saturation threshold 


\begin{tabular}{|c|c|c|c|c|c|c|}
\hline \multirow[t]{2}{*}{ Date } & \multicolumn{5}{|l|}{ 1/30/04 } & \multirow[t]{3}{*}{ Material: Optical Grade Spectralon } \\
\hline & \multicolumn{2}{|c|}{ Whole Beam } & \multicolumn{2}{|c|}{ Central $60 \%$} & \multirow[b]{2}{*}{$\begin{array}{c}\# \\
\text { shots }\end{array}$} & \\
\hline site & $\begin{array}{l}\text { F avg } \\
{\left[\mathbf{J} / \mathrm{cm}^{2}\right]}\end{array}$ & $\begin{array}{c}\mathbf{F} \text { pk } \\
{\left[\mathbf{J} / \mathbf{c m}^{2}\right]}\end{array}$ & $\begin{array}{c}\text { F avg } \\
{\left[\mathrm{J} / \mathrm{cm}^{2}\right]}\end{array}$ & $\begin{array}{c}\mathbf{F} \mathbf{p k} \\
{\left[\mathbf{J} / \mathbf{c m}^{2}\right]}\end{array}$ & & \\
\hline 1 & 2.6 & 4.8 & 2.8 & 4.8 & 1 & beam foot print visible \\
\hline 2 & 1.5 & 3.1 & 1.6 & 3 & 5 & $\begin{array}{l}\text { beam foot print visible after shot } 1 \text {, about } 3 \\
\text { min between first } 4 \text { shots and } 1 \text { min between } \\
4 \& 5\end{array}$ \\
\hline 3 & 1.1 & 2.3 & 1.1 & 2.3 & 10 & $\begin{array}{l}\text { beam foot print almost not detectable, } 12 \text { secs } \\
\text { between shots }\end{array}$ \\
\hline 4 & 2.5 & 5.6 & 2.5 & 5.6 & 10 & severe damage, 12 secs between shots \\
\hline
\end{tabular}

Table 1. Damage test results for optical grade Spectralon (internal memo by Mary Norton, Gene Donohue, and Bill Hollingsworth at LLNL). 